\title{
Clinicomicrobiological profile of infective endocarditis in a tertiary care center of Nepal
}

\author{
Ghimire $\mathrm{M}^{1}$, Karki $\mathrm{P}^{1}$, Khanal B², Acharya $\mathrm{P}^{1}$, Sharma SK${ }^{1}$, Pahari Bishnu ${ }^{1}$ \\ Department of Internal Medicine', Department of Microbiology ${ }^{2}$, B.P. Koirala Institute of Health Sciences, Dharan, Nepal.
}

\section{Abstract Background}

Infective endocarditis is a common problem and data regarding its clinical and microbiological pattern from developing countries are sparse. We studied clinical features and the microbiological pathogens in patients with Infective Endocarditis in our Hospital.

\section{Objective}

To study the clinical profile and microbiological pathogens involved in patients with Infective Endocarditis, admitted under Department of Internal Medicine of B.P Koirala Institute of Health Sciences.

\section{Materials and methods}

A total of 54 patients with history of fever and underlying heart disease were studied. It was a hospital based cross sectional descriptive study done in patients with infective endocarditis presented to us from March 2007 to February 2008 in B.P Koirala Institute of Health Sciences.

\section{Results}

Out of 54 patients, 11 (20.4\%) had Dukes definite IE. The male: female ratio was 1.2:1. The mean age of the study group was 27.3 years (range=16-55). In IE group, fever was present in $100 \%$ cases $(n=11)$ as it was the inclusion criteria of the study, followed by SOB $81.8 \%(n=9)$. History of antibiotic therapy prior to the presentation was present in $36.7 \%(n=4)$ patients. Pallor was the most common sign $63.6 \%(n=7)$. Splenomegaly was seen in $18.2 \%(n=2)$. Anaemia $(H b<10 g m \%)$ in $36.4 \%(n=4)$ and microscopic haematuria in $72.3 \%(n=8)$ cases. Blood culture positivity was seen in $36.4 \%$. The most common pathogens were Staphylococcus aureus in $27.3 \%(n=3)$ and Acinetobacter species in $9.1 \%$ $(n=1)$.

\section{Conclusion}

In our study we found that the clinical spectrum of IE was different from the west in that the majority of patients being young in our study. However, RHD still is the commonest underlying heart disease and Staphylococcus aureus being the commonest isolate.

\section{Key words: Infective endocarditis, rheumatic heart disease, staphylococcus aureus.}

\section{Correspondence}

Dr Madhav Ghimire

Email: madhavghimirenp@yahoo.com 


\section{Introduction}

Infective endocarditis (IE) remains a serious cardiac problem despite the availability of improved diagnostic and therapeutic facilities. Recent studies from the west have shown remarkable changes in the spectrum of IE. ${ }^{1,2,30}$ These changes are attributable to changes in substrate population, introduction of more accurate diagnostic criteria (Duke's criteria), better diagnostic facilities, availability of a range of antibiotics and aggressive surgical approach. Data regarding the pattern and outcome of infective endocarditis from developing countries are sparse. ${ }^{3,4}$ We therefore, undertook this study to determine the clinical characteristics and microbiological profile of patients with Infective endocarditis attending our hospital.

\section{Materials and methods}

A hospital based cross sectional descriptive study was done in patients from March 2007 to February 2008 in B.P Koirala Institute of Health Sciences. Consents were taken from all the participants. Ethical clearance obtained from ethical committee.

Inclusion criteria - The patients with systolic and diastolic murmur on clinical examination in association with fever were included in the study. In this study, fever of 3 days duration was taken and fever was defined as morning A.M temperature $>37.2^{\circ} \mathrm{C}\left(98.9^{\circ} \mathrm{F}\right)$ or evening P.M temperature of $>37.7^{\circ} \mathrm{C}\left(99.9^{\circ} \mathrm{F}\right)$ in oral cavity. ${ }^{5}$

\section{Exclusion criteria-}

Subjects were excluded from the study if they were found to have normal valve in echocardiography.

A total 54 subjects meeting the inclusion criteria were subjected to further evaluation with blood culture and echocardiography. Blood samples were taken from all the patients. Standard techniques were used for the isolation and identification of the pathogen. ${ }^{6}$ Out of 54 patients, 11 patients met the Duke's criteria of Infective endocarditis. Data of Infective endocarditis patients were entered on MS Excel sheet and were analysed with SPSS 15.0 version. The mean and standard deviation of different variables were calculated. For qualitative data chi square test was used and for quantitative data mean was compared in between dependent and independent variables.

\section{Results}

The median age of the study group was 32 years (range=16-55). There were 5 female and 6 male patients. Male to female ratio was 1.2. Fever being the inclusion criteria of the study, was present in all 11 patients followed by shortness of breath in 9 patients, anorexia in 9 patients, malaise in 7 patients, cough in 6 patients, palpitations in 5 patients, swelling of body in 5 patients, myalgia in 4 patients, and chest pain in 2 patients. Weight loss was seen in 2 patients. While none of the patients in IE group had easy fatigability, hemiplegia, seizure and syncope. On clinical examination pallor was the most common sign in 7 patients, Cyanosis and clubbing were seen in one patient. Splenomegaly was found in 2 patients. Embolic events were seen in 2 patients. Dental caries were seen in1 patient. Petechial haemorrhage was seen in 2 patients. Oedema was seen in 6 patients and icterus in 4 of the patients.

History of RHD was present in 2 patients and only 2 patients were under Penicillin prophylaxis. One patient was intravenous drug abuser and 4 patients were smoker. History of antibiotic therapy prior to the presentation was present in 4 patients. RHD was present in 9 patients. Congenital heart disease was present in one of the patient. Right sided endocarditis (IV drug abuser) seen in 1 patient. Degenerative and prosthetic valve diseases were not seen in any of the patient. No patients in the IE group had undergone recent dental or invasive procedure in last 2 weeks prior to the presentation. None of the patients had a history of prior IE.

Anaemia ( $\mathrm{Hb}<10 \mathrm{gm} \%)$ was present in 4 cases while leucocytosis and microscopic haematuria 
were present in 8 cases and 3 cases, respectively. (Table1).

Combined mitral, tricuspid and aortic valve pathology was the most common valvular pathology present in 4 cases. Similarly combined mitral and aortic valve pathology was seen in 4 cases. Combined mitral and tricuspid valvular pathology was seen in 2 patients. Isolated tricuspid valve involvement was seen in only 1 patient. None of the patients had prosthetic valve IE.

Blood culture positivity was seen in $36.4 \%$ (i.e. out of 11 diagnosed IE patients, 4 had blood culture positivity). The most common pathogens were: Staphylococcus aureus in $27.3 \%(n=3)$, and Acinetobacter species in $9.1 \%(n=1)$. Prior antibiotic treatment was received by $36.7 \%$ $(n=4)$ patients. (Table 2 )

\section{Discussion}

Infective endocarditis is an important cause of morbidity and mortality among cardiac patients. The reported studies of IE from developing countries are very less. ${ }^{4,7}$

An overall increase in the age of affected patients has been a notable feature of IE in last 30 years $^{8}$ possible reasons for this trend are the decline in incidence of rheumatic fever in the developed world, increasing numbers of adults rendered susceptible to IE by degenerative heart disease associated with old age and prolonged survival of patients with valvular disease due to improved medical and surgical treatment. ${ }^{8}$

Table 1. Clinical profile of Infective endocarditis patients.

\begin{tabular}{|c|c|c|}
\hline \multicolumn{3}{|c|}{ Clinical Profile } \\
\hline \multicolumn{2}{|c|}{ Mean age: } & $\begin{array}{l}27.3 \text { years } \\
\text { (range }=16-55)\end{array}$ \\
\hline \multirow{2}{*}{ Sex } & Female: & $45.5 \%(n=5)$ \\
\hline & Male & $54.5 \%(n=6)$ \\
\hline \multicolumn{2}{|c|}{ Male to female ratio } & 1.2 \\
\hline \multicolumn{2}{|c|}{ History of RHD } & $18.2 \%(n=2)$ \\
\hline \multicolumn{2}{|c|}{ History of IE } & None \\
\hline \multicolumn{2}{|c|}{ Patient under penicilline prophylaxis } & $18.2 \%(n=2)$ \\
\hline \multicolumn{2}{|c|}{ History of antibiotic therapy prior to presentation: } & $36.7 \%(n=4)$ \\
\hline \multicolumn{2}{|c|}{ History of IVDU } & $9.1 \%(n=1)$ \\
\hline \multicolumn{2}{|c|}{ Presence of RHD } & $81.8 \%(n=9)$ \\
\hline \multicolumn{2}{|c|}{ Presence of CHD } & $9.1 \%(n=1)$ \\
\hline \multicolumn{2}{|c|}{ Right sided endocarditis (IV drug abuser) } & $9.1 \%(n=1)$ \\
\hline \multicolumn{2}{|c|}{ Recent dental or invasive procedure in last 2 weeks } & None \\
\hline \multicolumn{2}{|c|}{ Degenerative aortic valve disease } & None \\
\hline
\end{tabular}

Table 2. Blood culture and pathogens.

\begin{tabular}{|l|l|}
\hline Blood culture positivity: & $36.4 \%(n=4)$ \\
\hline Most common pathogens were: Staphylococcus aureus & $27.3 \%(n=3)$ \\
\hline Acinetobacter species & $9.1 \%(n=1)$ \\
\hline
\end{tabular}


Majority of patients in IE group, $72.3 \%(n=8)$ in our study were below 36 years, this is similar to all previous studies of IE from India. ${ }^{4,7}$ But this is in contrast to the western studies where majority of patients had presented beyond the fourth decade. ${ }^{9,10}$ This can be due to the fact that RHD continues to be a significant problem in developing world which usually presents at younger age. ${ }^{11}$ More than half of our patients of IE had RHD as underlying heart disease. Increasing age of patients of IE in west is attributable to; markedly decrease frequency of RHD, ${ }^{12}$ longer survival of patients with valve disease and CHD because of readily available modern medical and surgical therapy, increased incidence of degenerative valvular disease, ${ }^{2}$ aging of the population in general ${ }^{9,13}$ and more frequent use of invasive central vascular catheters which are associated with an increase in nosocomiallyacquired endocarditis. There was a significant rise in the age of the patients during the 1940s ,1950s and $1960 \mathrm{~s}^{14}$ and studies covering the time periods $1968-1973,{ }^{15} 1973-1984^{16}$ and $1970-1977$ ${ }^{17}$ found the mean age to lie within the sixth decade. Our findings suggest that there has been no further increase in the age of the patients and concur with two recent studies. ${ }^{18}$ In contrast, two other studies ${ }^{19,}{ }^{20}$ suggests a continued increase in age of patients. The median age of patients with endocarditis from western countries in the pre-antibiotic era was about 35 years, and in subsequent decades the age at presentation has gradually increased to $>50$ years. ${ }^{9,21}$

RHD was the commonest underlying heart disease in our study that is similar to other studies from India. ${ }^{3}$ But this is in contrary to western reports and is explained by decreased incidence of rheumatic fever there..$^{12}$ In our study RHD was predisposing condition in higher no of IE patients than the figures reported in most recent series. ${ }^{22,}$ ${ }^{23}$ Right sided IE secondary to intravenous drug abuse is showing an alarming increase in west. ${ }^{24}$ Our study has also shown $9.1 \%$ of intravenous drug abuse that was associated with right sided endocarditis. The proportion of patients with intravenous drug abuse varied between $1 \%$ and

\section{$67 \%$ in reported series. ${ }^{2,13}$}

Patients who were receiving regular penicillin prophylaxis and who developed infective endocarditis were only $18 \%$. So the reason for others to develop infective endocarditis may be due to irregular penicillin prophylaxis. All patients improved with the treatment.

Blood cultures have been reported to be positive in more than $90.0 \%$ patients of IE in western series. ${ }^{13,25}$ But in our study only $36.4 \% \quad(n=4)$ episode of IE were culture positive. This is in accordance to previous studies in developing world, which have reported culture positivity in 21-47\% episodes. ${ }^{4,7,26}$ Prior antibiotic therapy may be the important reason for high culture negativity in our series, since a high proportion of culture negative IE patients had received antibiotic therapy before admission to our hospital [36.4\% $(n=4)]$. S. aureus have been reported as etiological agents in $13-49 \%$ of cases of native valve endocarditis. ${ }^{27}$ However in our study Staphylococcus aureus was isolated in $27.3 \%(n=3)$ of patients and $9.1 \%(n=1)$ of patients had unusual gram negative Acinetobacter species isolate. There were no growths of fastidious microorganisms such as the HACEK group. (Haemophilus aprophilus/paraphrophilis, Actinobacillus actinomycetemcomitans, Cardiobacterium hominis, Eikenella corrodens, Kingella kingae). Since only $36.4 \%$ of all episodes of IE are culture positive result in our series; the relative contribution of these microorganisms seems to be much less than reported in the literature. One of the major differences in our results from recently published studies is the low rate of culture positivity. Failure to culture the organism in IE may result from infection with highly fastidious bacteria or nonbacterial microorganisms, or from the administration of antimicrobial agents before blood cultures are obtained..$^{28}$ As $50 \%$ of our patients had previous use of antibiotics, we believe this was the main reason for our high number of culture negative cases. This is consistent with previous reports in which the number of patients with culture negative endocarditis who had received 
antibiotics in the 2 weeks prior to diagnosis was found to be as high as $60 \% .{ }^{1,29}$ Some IE pathogens such as HACEK organisms, nutritionally variant streptococci, Brucella and Bartonella species, some fungi, and Coxiella burnetti are difficult to isolate from blood cultures. ${ }^{28}$

Recent large western series have reported a change in the microbial spectrum. Staphylococcus aureus has been reported as the commonest microorganism with relative decline in streptococcal endocarditis. ${ }^{19}$ This is attributable to aging population, rising drug abuse, patients with prosthetic valves and increasing frequency of nosocomial and iatrogenic endocarditis. Similarity to the west studies, Staphylococcus was the commonest isolates $27.3 \%$ in our study. We had expected the streptococci viridans to come out as the common isolate as our population at risk is still young patient with RHD. Hence we need to consider, is the change in microbiological pattern being seen in west also going on in our part of the world?

The limitation of the study was the sample size which was small and some of the sick patient may not have reached the hospital. Many patients had prior antibiotic intake, which might have resulted in low culture positivity seen in our study. Despite these shortcomings, this study reflects the general picture of infective endocarditis at a tertiary hospital in a developing country and it shows the differences in predisposing factors and etiological agents.

\section{Conclusion}

Spectrum of IE in our country is different from the west, but quite similar as reported from developed countries about 40 years ago. It occurs in relatively younger population and RHD being the commonest underlying heart disease. Despite RHD being the commonest underlying heart disease, Staphylococcus aureus was the commonest pathogen isolated. Currently IE is a polymorphic disease that challenges the clinician, requiring management by a multidisciplinary team to reduce the mortality.

\section{Recommendation}

More work is necessary to better identify which patients are at risk for developing IE and its complications. Our study has shown encouraging results regarding the changing microbial pattern in IE in our setup, which is similar to western study but on the background of RHD being the common underlying heart disease which is again contrary to recent western studies. So, we need a large multicentre study to reflect the real epidemiological, microbiological pattern of IE in our country. Moreover the study results emphasize the need to conduct both close local epidemiological surveillance and multicentre studies with global view of IE to assist in the prevention, diagnosis, and management of this complex disease in order to allow more optimal management of suspected endocarditis cases in the future, it seems to be necessary to improve education of both patients at risk of developing IE and clinicians about the importance of blood cultures in the diagnosis of IE and rational use of antibiotics.

\section{Acknowledgements}

All patients in our research work and staffs of Department of Internal Medicine, BPKIHS, Dharan.

\section{References}

1. Kaye D. Changing pattern of Infective Endocarditis. Am J Med.1985; 78: 157-62.

2. Netzer ROM, Zollinger E, Seiler $C$, et al. Infective endocarditis: Clinical spectrum, presentation and outcome, An analysis of 212 cases 1980-1995. Heart. 2000; 84: 25-30.

3. Chaudhary R, Grover A, Varma J, et al. Active infective endocarditis observed in an Indian hospital 1981-1991. Am J Cardiol. 1992; 70:1453-8.

4. Jalal S, Khan KA, Alai MS, et al. Clinical spectrum of infective endocarditis; 15 
years experience. Indian Heart J. 1998; 50:516-9.

5. Charles AD, Jeffrey AG. Fever and Hyperthermia. In: Harrisons's principle of Internal Medicine, 16th ed. New York: McGraw- Hill Medical Publishing Division: 2005; 731-40.

6. Murray PR, Baron EJO, Pfaller MA, et al. Manual of clinical Microbiology, 6th ed. Washinton: ASM press, 1995.

7. Datta BN, Khatri HN, Bidwai PS et al. Infective endocarditis at autopsy in Northern India- a study of 120 cases. Jrn Heart J. 1982; 23:329-37.

8. Cherubin CE, Neru HC .Infective endocarditis at Presbyterian Hospital in New York City from 1938-1967. Am J Med. 1971; 51:83-96.

9. Awadallah SM, Kavey RW, Byrum CJ, et al. The changing pattern of infective endocarditis in childhood. Am J Cardiol. 1991; 68:90-4.

10. Grover A, Dhawan A, lyenger SD. Epidemiology of rheumatic fever and rheumatic heart disease in a rural community in Northern India. Bull World Health Organ. 1993; 71:59-66.

11. Gordis L. The virtual disappearance of rheumatic fever in United States: lessons in the rise and fall of disease. Circulation. $1985 ;$ 72:1155-62.

12. Pomerance A. Cardiac pathology in elderly. Cardio vasc Clin. 1981; 12:9-54.

13. Bayer AS, Ward JI, Ginzton LE, et al . Evaluation of new clinical criteria for diagnosis of infective endocarditis. Am J Med. 1994; 96:211-9.

14. Nunley DL, Perlman PE. Endocarditis: changing trends in epidemiology, clinical and microbiologic spectrum. Post grad Med. 1993; 93:235-47.

15. Lien EA, Solberg CO, Kalager T. Infective endocarditis 1973-1984 at the Berben University Hospital: Clinical features, treatment and prognosis. Seand J Infect Dis. 1988; 20: 239-246.

16. Tornos MP, Olona M, Permanyer-Miralda $\mathrm{G}$, et al .Is the clinical spectrum and prognosis of native valve endocarditis in non addicts changing? Eu Heart J. 1995; 16:1686-91.

17. Pelletier LL, Petersdorf RG. Infective endocarditis: a review of 125 cases from the University of Washington Hospitals, 1963-1972. Medicine. 1977; 56:287-313.

18. Watanakunakorn C, Burkert T. Infective endocarditis at a large community teaching hospital.1980-1990.A review of 210 episodes .Medicine. 1993; 72:90102.

19. Van der Meer JT, Thompson J, Valkenburg $H A$, et al . Epidemiology of bacterial endocarditis in the Netherlands. Arch Intern Med. 1992; 152:1863-8.

20. Watanakunakorn C. Changing epidemiology and newer aspects of infective endocarditis. Adv Intern Med. 1977; 22:21-47.

21. Cecchi E, Parrini A, Chinaglia F, et al. New diagnostic criteria for infective endocarditis: a study of sensitivity and specificity. Eur Heart J. 1997; 18:1149-56.

22. Lamas CC, Eykyn SJ. Suggested modifications to the Duke criteria for the clinical diagnosis of native valve and prosthetic valve endocarditis: analysis of 118 pathologically proven cases. Clin Infect Dis. 1997; 25:713-9.

23. Siddiq S, Missri J, Silverman DI. Endocarditis in an urban hospital in the 1990s. Arch Intern Med. 1996; 156:24548.

24. King JW, Nguyen VQ, Conrad SA. Results of a prospective state wide reporting system for infective endocarditis. Am J 
Med Sci. 1988; 295:517-27.

25. Sandre RM, Shafiran SD. Infective endocarditis: review of 135 cases over 9 years. Clin Infect Dis. 1996; 22:276-86.

26. Khanal B, Harish BN, Sethuraman KR, et al. Infective endocarditis: report of prospective study in an Indian hospital. Tropical Doctor. 2002; 32:83-5.

27. Tariq M, Alam M, Munir G, et al. Infective endocarditis: a five-year experience at a tertiary care hospital in Pakistan. International Journal of Infectious Diseases. 2004; 8: 163-70.

28. Hoen B, Selton-SC, Lacassin F, et al. Infective endocarditis in patients with negative blood cultures: analysis of 88 cases from a one-year nationwide survey in France. Clin Infect Dis. 1995; 20: 501-6.

29. Tunkel AR, Kaye D. Endocarditis with negative blood cultures. $N$ Engl J Med. 1992; 326:1215-7.

30. Durack DT, Lukes AS, Bright DK. Duke endocarditis service. New criteria for diagnosis of infective endocarditis: utilization of specific echocardiographic findings. Am J Med. 1994; 96:200-9. 